\title{
55 \\ AN APPROACH TO CONFORMANCE TESTING OF MIB IMPLEMENTATIONS
}

\author{
Michel Barbeau \\ Université de Sherbrooke \\ Dépt. de mathématiques et d'informatique \\ Sherbrooke, Québec \\ Canada J1K 2R1 \\ Tel. +1-819-821-7018 \\ E-mail:barbeau@dmi.usherb.ca
}

\author{
Behcet Sarikaya \\ University of Aizu \\ Computer Communications Lab. \\ Tsuruga, Ikki-machi, Aizu-Wakamatsu \\ Fukushima, Japan 965-80 \\ Tel. +81-242-37-2559 \\ E-mail: sarikaya@rsc.u-aizu.ac.jp
}

\begin{abstract}
A methodology is presented to test the conformity of managed nodes to network management standards in the SNMP framework. The first phase of the methodology consists of an object-oriented modeling of the managed node using class diagrams and SDL-92 language. The second phase takes the abstract model to systematically generate test suites. The approach is based on ISO's conformance testing methodology. Test cases are expressed in the Tree and Tabular Combined Notation (TTCN). The approach is illustrated with a recently developed Management Information Base (MIB) for the management of ATM permanent virtual links.

Keywords

Management Information Base, Simple Network Management Protocol, ASN.1, SDL-92, Class diagrams, Conformance Testing, Abstract Test Suites, TTCN.
\end{abstract}

\section{INTRODUCTION}

Presently there are two main frameworks for network management, namely, the OSI and Internet Engineering Task Force (IETF) frameworks. IETF has developed a simple view of network management called the Structure of Management Information (SMI) and Simple Network Management Protocol (SNMP) [9]. The approach presented in this paper has been done for IETF framework, also known as the SNMP framework.

A network in the SNMP framework is made of several managed nodes and at least one management station. Every managed node has several managed objects. Managed objects are abstractions of data processing and data communication resources, such as routing tables and counters. They represent the management view of network resources which can be physical or conceptual in nature. Managed objects and management protocol data units (PDU) constitute the management information. Management information representation in SMI is done using a subset of ASN.1 [6] with macros.

Managed objects are grouped in Management Information Bases (MIBs). MIBs are maintained by every managed node. There are several MIB models that serve different purposes and are attached to different technologies. For instance, MIB-II has been defined to manage TCP/IP networks [9] and ATOMMIB to manage permanent circuits of Asynchronous Transfer Mode (ATM) networks [1].

In this paper we develop a test design methodology for testing conformity of managed nodes to network management standards published by IETF and known as RFCs. The 
paper continues in Section 2 where a technique is introduced for reverse-engineering the descriptions of the managed nodes and obtaining a precise behavior model in SDL-92. In Section 3 the test design methodology is detailed. In Section 4 we discuss use of the SDL-92 specification to generate test cases. The approach is illustrated with the traffic description parameters group of the ATM MIB. Finally, Section 5 presents some concluding remarks.

\section{REVERSE-ENGINEERING OF MIBS}

Reverse-engineering is defined as taking something at a level of abstraction and deriving from it something at a higher level of abstraction. In the SNMP framework, MIBs are described with ASN.1, for the structural aspects, and natural language, for the behavioral aspects.

\subsection{Structure of Management Information}

Managed objects are data structures maintained by every managed node. In SMI, values of managed objects are of various ASN.1 types. In addition, every object has a distinct name of type OBJECT IDENTIFIER. For instance, managed objects in the Internet network all have the following common prefix:

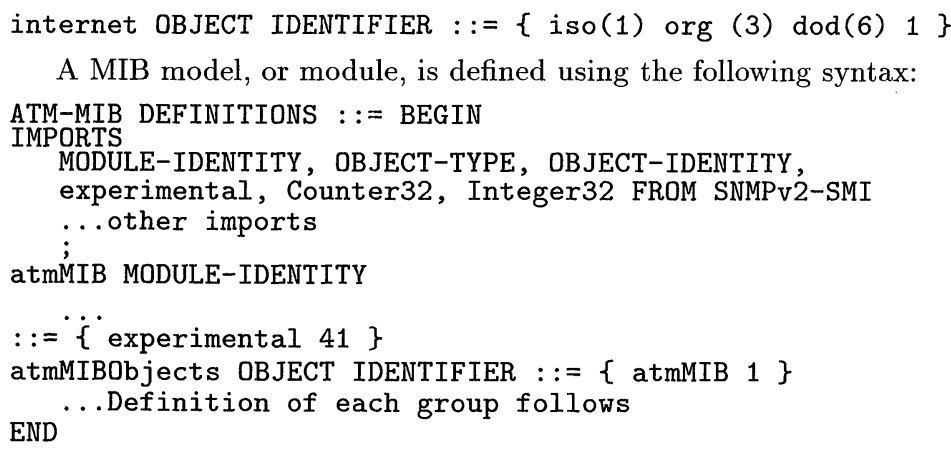

A MIB model, or module, is defined using the following syntax:

Every module has a name, e.g., ATM-MIB. A module can import definitions from other modules, e.g., MODULE-IDENTITY, OBJECT-TYPE, OBJECT-IDENTITY, experimental, Counter32, and Integer32 are imported from module SNMPv2-SMI. Most of the commonly used definitions have already been declared in the module SNMPv2-SMI. In the above, the MODULE-IDENTITY macro is used to define the module's identity as experimental 41 and document its revision history. Managed objects are defined within logical groups. Each group corresponds to one aspect of the system, e.g., a protocol layer.

Managed objects within groups are defined using the OBJECT-TYPE macro. Macros have symbolic expansion capability such as Backus-Normal Form (BNF) rules devised for describing the syntax of programming languages. Macros have several clauses. Clause SYNTAX defines the data type of the object. Clause MAX-ACCESS specifies the level of access such as read-create or not-accessible. Clause STATUS serves to create versions of MIBs. Finally, clause DESCRIPTION introduces a textual description of the managed object.

A MIB can be seen as a collection of simple (scalar) and more complicated tabular objects. Tabular objects will be explained using ATM MIB. A tabular object called interface configuration table is defined in the ATM MIB as follows: 


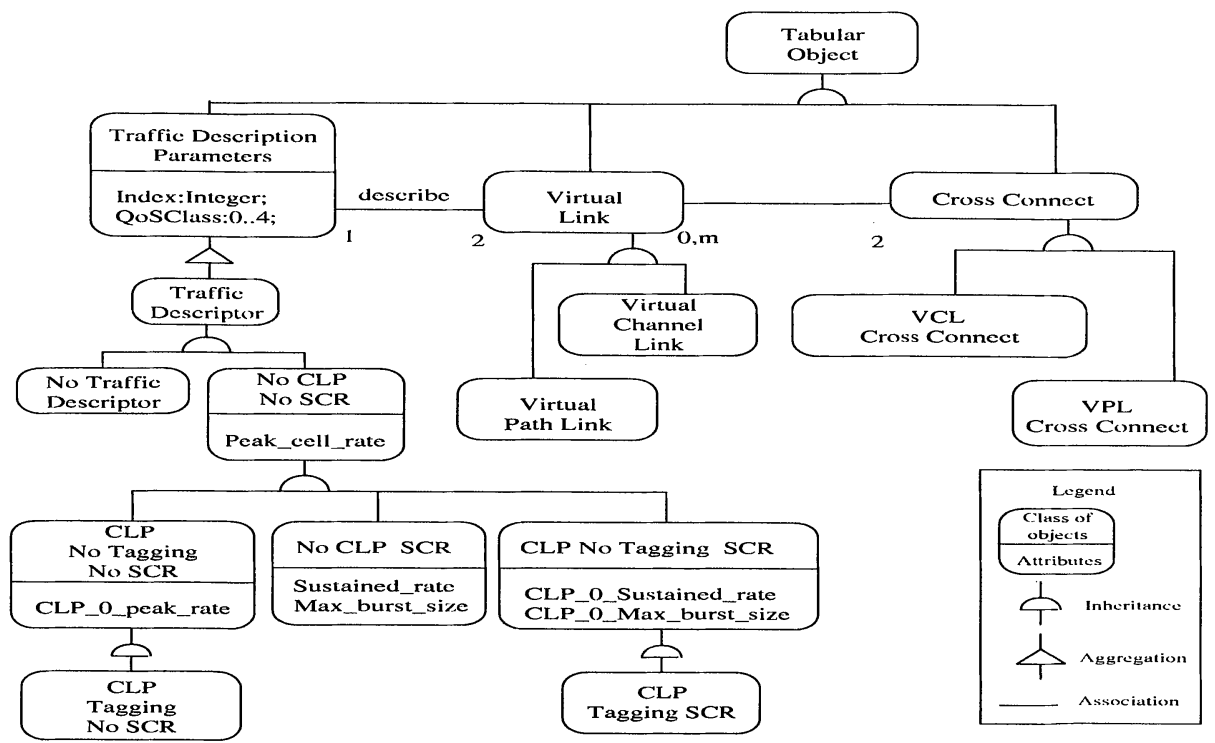

Figure 1: Class Diagram of ATM MIB

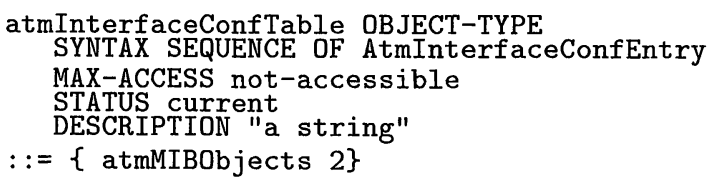

The table is defined as an unbounded list of values of type AtmInterfaceConfEntry, the rows of the table. AtmInterfaceConfEntry is defined as a record of values of various types. Such values are called columnar objects. In order to distinguish between different rows, an index (often of type INTEGER) is defined using the INDEX clause of the OBJECT-TYPE macro. The definition of atmInterfaceConfTable is completed as follows:

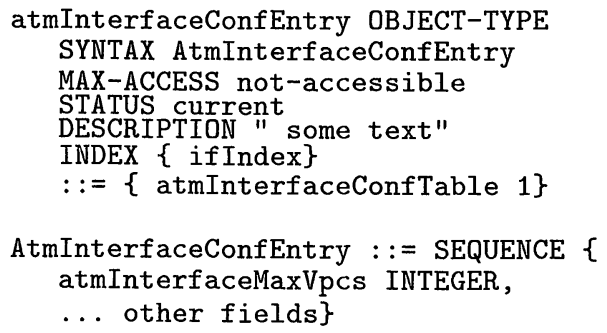




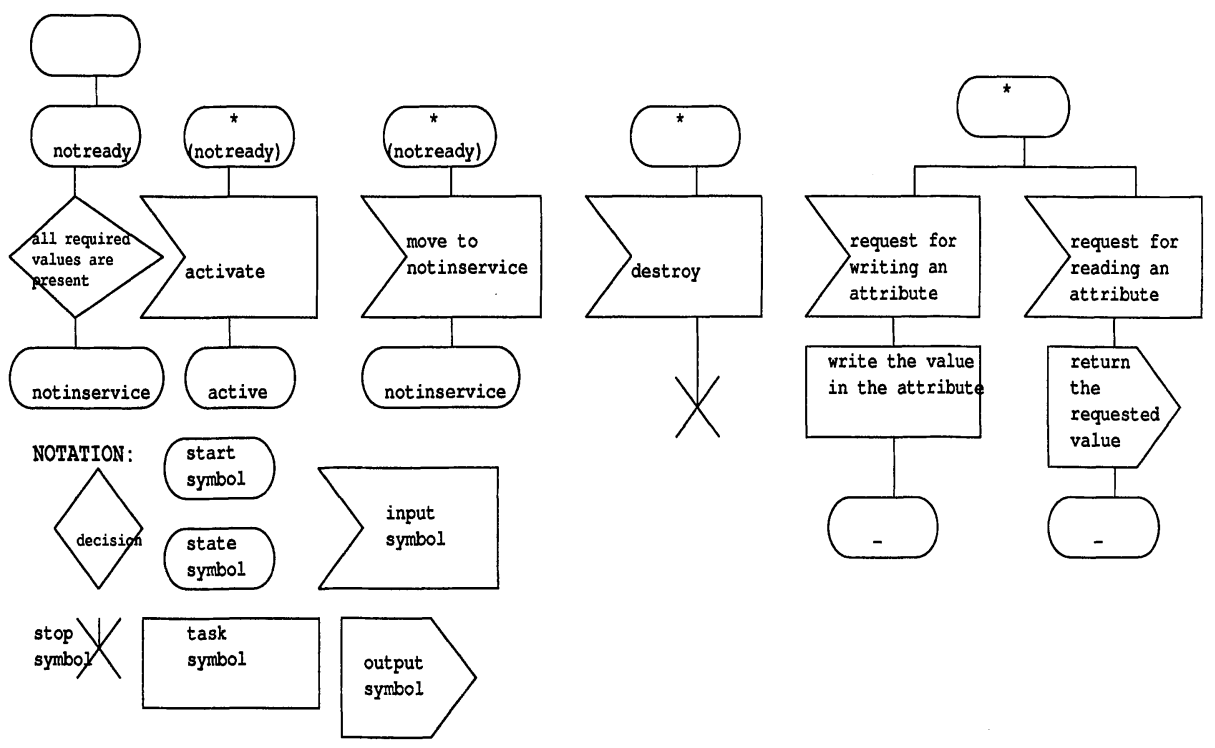

Figure 2: Tabular Object Process Diagram

\subsection{Methodology}

Our technique is based on class diagrams [3] and SDL-92. Class diagrams clearly show the structures to be tested and common aspects of these structures. SDL-92 specifications precisely define the behavior to be tested. Both are obtained by inspection of the ASN.1 managed object data structures, the accompanying textual description in the MIB RFCs, and SNMP protocol elements. Additional information about the system to be managed is also needed most of the time. For the example discussed in the paper, it was obtained from [5].

A class diagram shows classes of objects, subtyping relationships among classes (i.e., inheritance), containment relations (i.e., aggregation), and other associations among objects [3]. For every ASN.1 simple object type, such as Counter32, we define an object class. This class contains two attributes, one for storing the object value, such as integer, and another for storing the name of a particular object of this class, such as snmpStatsPackets. In addition, operations, such as increment, are defined to capture the semantics of the class.

Tables in the MIB are mapped to classes. Each row in a table is modeled as a class instance. Most of the fields of the table are mapped to attributes, unless they serve to subtype or establish relations between objects. A field of type OBJECT IDENTIFIER may serve to subtype the rows of a table. The values of the field identify the subtypes. Some of the other fields in the table may be applicable only for some subtypes. This is reflected in the class diagram as a superclass (representing the subtyping field) with as many subclasses as there are possible values for the field. Subtype specific fields are 


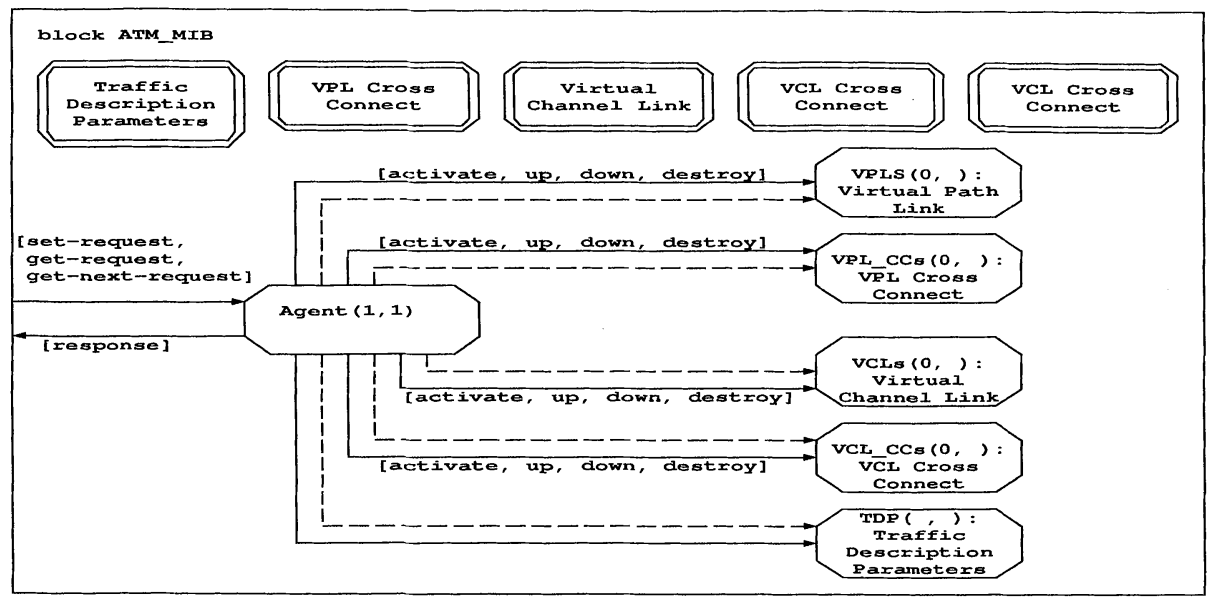

Figure 3: Block Diagram of ATM MIB

moved to the related subclasses. An aggregation relation from the superclass to the class representing the table is also created. Some fields may represent indexes in other tables. They are represented as relations among objects. Finally, superclasses are also introduced to put at on place definitions of attributes and relations common to several other classes.

In SDL-92 [4], behavior is described in terms of processes interacting with an environment. Processes consume signals and perform actions in return. The behavior of a process is modeled as an extended finite-state machine. SDL-92 allows definition of process types as well as reuse of types by inheritance. Processes can be organized into logical blocks.

Classes with behavior in the class diagram are mapped to SDL-92 process types in a block diagram. Communication and process creation relations are uncovered and also represented in the block diagram. Simple object types are static and behavior must be defined to capture the temporal dependencies between the operations, e.g., a gauge can be incremented only if its value is lower than its maximum value.

Rows of ASN.1 tables represent dynamic entities. In SMI, the states in which a row goes through during its life cycle is coded as an integer in a field of type RowStatus. Some of these values represent states of instances, some of them represent actions on the instances, and others represent both states and actions.

Procedures of the agent for creating and destroying rows for every kind of table must be specified in SDL-92. The procedure for a creation is initiated by a SNMP set-request PDU identifying a row in which the value createAndGo or createAndWait is written in its RowStatus field. Value createAndGo is used for single step creation, i.e., all the values of the fields of the row are provided in a single set-request PDU. Value createAndWait is for negotiated creation during which values of components are written one after the other allowing detailed error checking. The procedure for destroying a row is initiated by a set-request PDU identifying the row in which the value destroy is written in its RowStatus field.

The behavior of a process modeling a row in a table, called Tabular Object, is conceptualized as the following SDL-92 process type definition: 


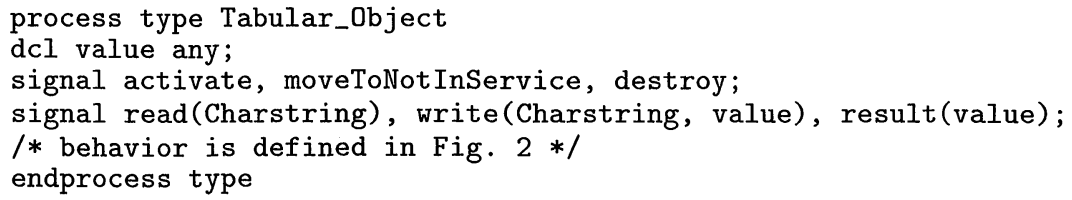

The behavior of a tabular object is specified in the graphical form of SDL-92 (Fig. 2). There are three states in Fig. 2: active, notReady, and notInService. Initially, an object is created and put in the notReady state. In the notReady state, as well as in other states, the agent can receive a request for writing or reading the value of a field (the two rightmost transitions in the figure). There are values of attributes that are required to be present by the agent. When all the required values are present, the object spontaneously moves to the notInService state (the leftmost transition). In this state the agent can put the object in the active state by sending an activate command which is modeled as a SDL signal (the second transition from left). An active object can be put in the notInService state by signal moveToNotInService (the third transition from left). From any state the object can be destroyed by sending a signal destroy (the fourth transition from left). The definition of Tabular Object can be reused by inheritance for any class of objects modeling tables.

\subsection{ATM MIB Example}

In an ATM network there are two kinds of virtual connections, namely, switched virtual connections and permanent virtual connections. Permanent bi-directional virtual connections are the main entities considered under this SNMP framework. There are two categories of permanent virtual connections, that is, permanent virtual channel connections (VCCs) and permanent virtual path connections (VPCs). A VCC is carried by a VPC. Conversely, a VPC can carry several VCCs. A VCC/VPC is made of virtual channel/path link's (VCL/VPL's) cross connected together.

Fig. 1 shows the main classes and relations of the ATM MIB. The class Tabular Object defines properties, i.e., attributes and behavior, common to traffic description parameters, virtual links, and cross connections. The class Virtual Link is abstract and further specialized into the subclasses Virtual Channel Link and Virtual Path Link, similarly for class Cross Connect. Classes Traffic Description Parameters, Virtual Channel Link, Virtual Path Link, VCL Cross Connect, and VPL Cross Connect originally appear as tables in Ref. [1].

Instances of the class Cross Connect model cross connections (CCs) between virtual links. There are two subclasses because a cross connect may be either between two VCLs or two VPLs. Note that between the class Virtual Link and Cross Connect there is the association is connected by linking the cross connected virtual links. Such an association is originally coded in SMI as two fields in rows storing two virtual channel identifiers (virtual path identifiers). VCLs and VPLs may be cross connected according to three topologies, i.e. point-to-point, point-to-multipoint, and multipoint-to-multipoint. A point-to-point CC associates two VCL/VPLs and is modeled as one instance of the class Cross Connect associated with the corresponding two instances of the class Virtual Channel Link/Virtual Path Link. A point-to-multipoint CC associates a VCL/VPL with several other VCL/VPLs and is modeled as several instances of the class Cross Connect. Every instance models attachment of the single point VCL/VPL to one of the multipoint VCL/VPL. A multipoint CC is similarly modeled as several instances of the class Cross Connect. 


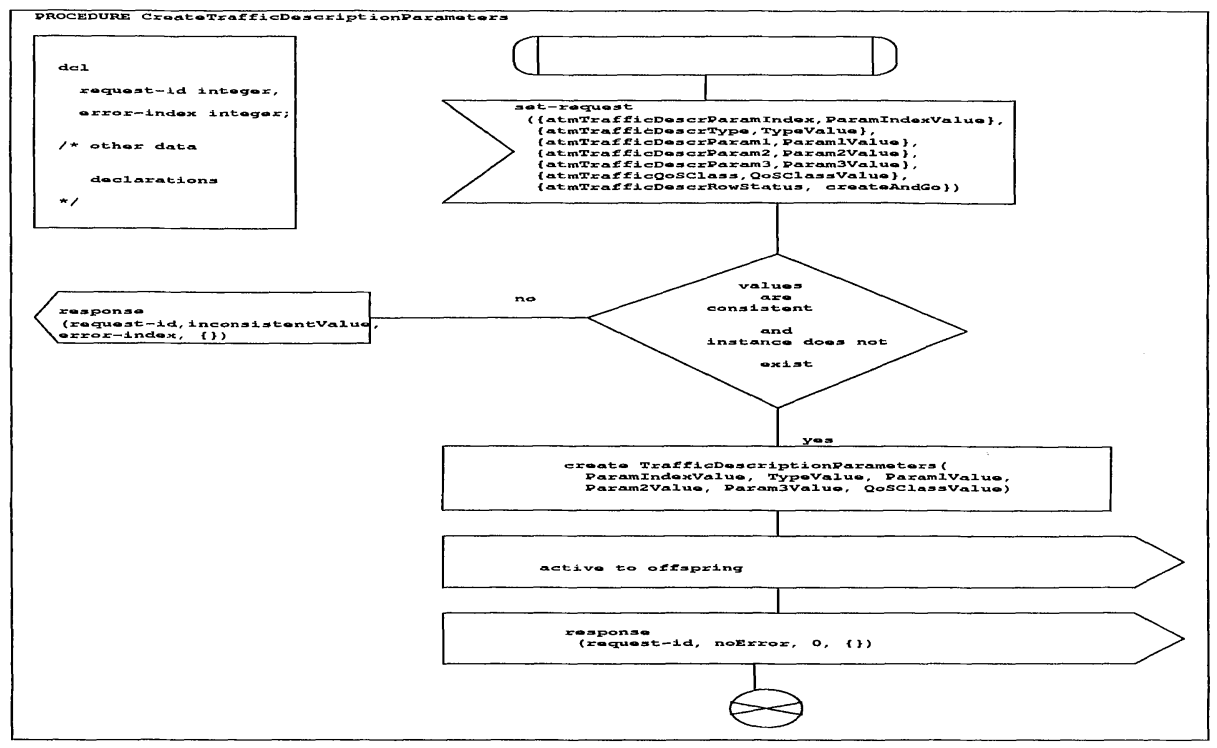

Figure 4: Traffic Description Parameters Creation Procedure

Bandwidth requirements of VCLs and VPLs are given by the users and described in terms of traffic description parameters. In Fig. 1, the class Virtual Link has an association to the class Traffic Description Parameters. The cardinality of this association is one-to-two because two sets of parameters are required to characterize the two traffic flow directions on a virtual link. An instance of class Traffic Description Parameters has two attributes, namely, Index and QoSClass. The attribute Index serves to identify the instances whereas the attribute QoSClass indicates the quality of service required by the connections. Class Traffic Description Parameters appears as a table in Ref. [1]. One of the fields in the rows of that table is of data type OBJECT IDENTIFIER. The values identify the seven possible ATM traffic descriptor types. This is modeled as a class with subclasses and an aggregation relation. That is, an instance of the class Traffic Description Parameters contains also an instance of the class Traffic Descriptor which have seven subclasses. In Fig. 1, acronym CLP stands for Cell Loss Priority and SCR stands for Sustained Cell Rate. In the ASN.1 representation of the MIB, attributes Peak_cell_rate and CLP_O_peak_rate are known under the names atmTrafficDescrParam1 and atmTrafficDescrParam2. Attributes (CLP_O_ $)$ Sustained_rate and (CLP_O_)Max_burst_size are known under the names atmTrafficDescrParam2 and atm TrafficDescrParam3.

We now discuss specification in SDL-92. The ATM MIB is encapsulated into the SDL92 block pictured in Fig. 3. Most classes from the class diagram of Fig. 1 are mapped to SDL-92 process types. Class Traffic Descriptor is not mapped to a process type because it has no behavior. Hereafter, we provide a specification in SDL-92 of a procedure that must be supported by the agent for handling requests for the creation of instances of class Traffic Description Parameters. 
Fig. 4 pictures creation of traffic description parameters of a VCL/VPL by a manager. The first action is the reception of a PDU set-request from the manager by the agent. The manager must provide an index (the identifier of the instance), a type (selected among the seven types that appear in Fig. 1), parameters describing the traffic characteristics and the quality of service class, and a row status value (which is createAndGo). The agent then decides if the traffic parameter values are consistent and makes sure that an object with the same index has not yet been created. In the affirmative, an instance of process type Traffic Description Parameters is created. Values of the set-request parameters are passed to the created process. The signal active is sent to the new object, a positive response $\mathrm{PDU}$ is returned to the manager and the procedure terminates. Otherwise, a response $\mathrm{PDU}$ with error status set to inconsistent Value is returned to the manager. The behavior of process type Traffic Description Parameters is as follows:

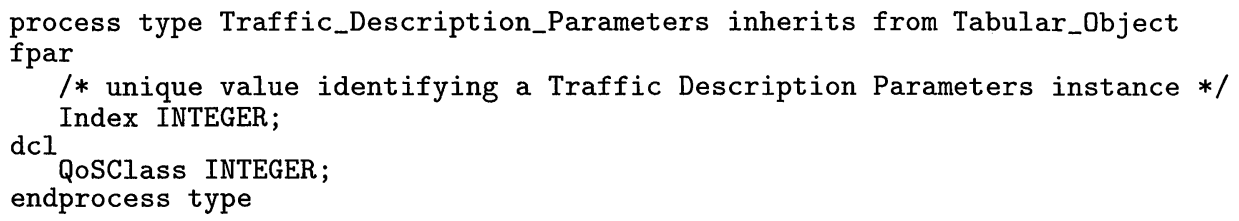

Because of inheritance, instances of process type Traffic Description Parameters behave like instances of process type Tabular Object. A complete specification of the ATM MIB is described elsewhere [2].

\section{ATM MIB CONFORMANCE TESTING}

In this section, we shortly review conformance testing of managed objects according to the OSI conformance testing methodology. Afterwards, the methodology is applied to the design of an abstract test suite for the ATM MIB.

\subsection{Conformance Testing of Managed Objects}

Conformance of implementations Under Test (IUT) of protocols for OSI are tested using standardized sets of test cases called Abstract Test Suites (ATSs). ATSs are formally specified in a language called Tree and Tabular Combined Notation (TTCN) [8].

There are difficulties in applying directly the OSI methodology [7] to the conformance testing of managed nodes since it is designed for protocol testing and direct access by PDUs to the managed objects is not possible. Direct access to the agent is possible by means of SNMP PDUs. The agent in its turn has direct access to the managed objects. Therefore managed object testing can be considered as service testing. The SNMP protocol interface is defined as the Point of Control and Observation (PCO).

In the SNMP framework, the expectations placed on a given MIB are defined using the MODULE-COMPLIANCE and OBJECT-GROUP macros. Conformance information about groups of objects, referred to in a MODULE-COMPLIANCE macro, is indicated using the OBJECT-GROUP macro. lows:

As an example, the group atmInterfaceConfGroup and its objects are defined as fol-

atmInterfaceConfGroup OBJECT-GROUP

OBJECTS \{ atmInterfaceMaxVpcs, atmInterfaceMaxVccs, -- other objects \} STATUS current 


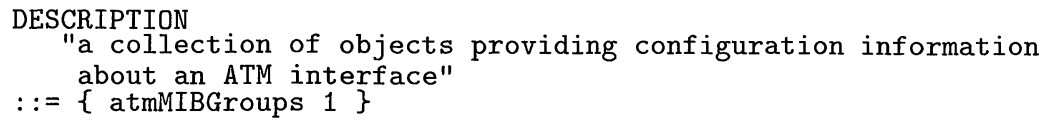

The SMI module-compliance information corresponds to the conformance statements in the OSI protocol standards. The SMI model incorporates a mechanism for the IUTs to claim their implementation capabilities. This makes it possible to select/deselect test cases, from an ATS, according to the claims of a given IUT. The SMI AGENT-CAPABILITIES macro is defined for this purpose. When this macro is invoked, zero or more modules are identified. For each module, the conformance groups implemented are listed. For each group, any variation is specified including the objects not implemented, not completely implemented or created only in conjunction with others. A capability statement is identified by an OBJECT IDENTIFIER. A management application can maintain a database of capability statements and then dynamically inspect these capability statements.

\subsection{Test Methodology}

In accordance with the OSI methodology, the ATS for the ATM MIB can be structured into four groups:

- Basic Interconnection Tests. This group contains a single test case whose purpose is to find out if an IUT supports SNMP. In this test, the tester sends a get-request PDU requesting the value of the managed object named sysDescr. If the tester receives a response, then the IUT passes this test and testing can be pursued.

- Capability Tests. The objective of this group is to establish whether or not a functional unit is available. If so, a representative of element of the functional unit is exercised. For MIB conformance testing, this involves checking the support of objects by the managed node.

- Valid Behavior Tests. This group contains test cases for each group defined in the ATM MIB. Valid behavior tests are designed for determining if the behavior assigned to each object has correctly been implemented. Valid behavior test design is further discussed in Section 4.

- Invalid Behavior Tests. The aim of this group is to test the responses of the IUT to syntactically or semantically invalid behaviors generated by the tester. The specification must include a description of how these exceptional cases must be treated. Syntactic encoding errors are normally captured by the ASN.1 encoding and decoding function of SNMP.

- Inopportune Behavior Tests. According to SNMP, any PDU can be sent at any time. Because of this, there will be no tests defined in this group.

\section{TEST GENERATION BASED ON SDL-92}

The process of obtaining a test suite from a formal specification can be viewed as the process of obtaining the behavior of an entity, called its conformance tester, exhibiting an inverse behavior. That is, inputs (outputs) to (from) the IUT are inverted to become the outputs (inputs) from (to) the tester. We call this process behavior inversion [10].

Test cases are selected such that all control and data flow paths in a SDL-92 specification are covered. The model of a SDL-92 specification is an extended finite-state 


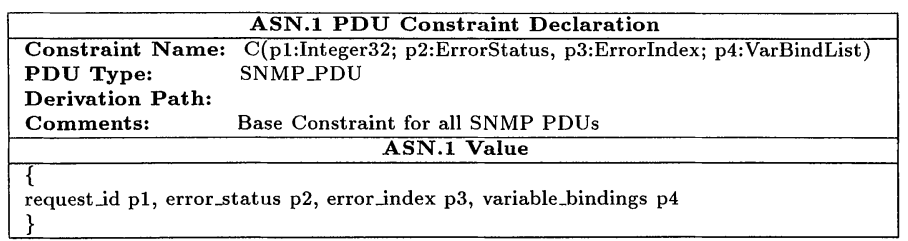

Table 1: Base Constraint

\begin{tabular}{|c|c|c|c|c|}
\hline $\mathrm{Nr}$ & Label & Behavior description & Cref & V \\
\hline 1 & & !set_request & Set_request_base & \\
2 & & START transmissionTimer & $\mathrm{C}(0, \mathrm{p} 2, \mathrm{p} 3,\{\})$ & \\
3 & LB & ?response & & \\
4 & & CANCEL transmissionTimer & & (P) \\
5 & & [p2=noError AND p3=0] & & FAIL \\
6 & & +POSTAMBLE & & \\
7 & & [NOT (p2=noError) AND (p3=0))] & & \\
8 & & ?trap & & INCONC \\
9 & & -> LB & & FAIL \\
10 & & ?TIMEOUT transmissionTimer & & \\
11 & & ?OTHERWISE & & \\
\hline
\end{tabular}

Table 2: Creation of Traffic Description Parameters Test Case 1

machine. The main testing strategy is state and transition coverage. Every distinct path, consisting of one or several transitions in the extended finite-state machine, is exercised by test cases. Each test case is assigned a distinct purpose, i.e., test of a given behavior following a certain path. Parameter values, of input and output signals of the test case, are selected according to the test purpose and such that predicates of the transitions in the test case are satisfied (in order to make the test case executable). Also, as a data flow testing strategy, parameter variation and combination of parameter values is employed.

Valid behavior test generation is illustrated in this section with an example taken from the ATM MIB. The valid behavior test group contains a subgroup for each MIB group. The example, presented in the sequel, is with a group called Traffic Descriptor Parameter.

Several test cases are needed for testing the valid behavior of group Traffic Descriptor Parameter. The behavior described in Fig. 4 is used to generate test cases. In the figure, one can identify two branches. The no branch of the first decision node defines an invalid behavior test. Therefore, in the valid behavior test cases the yes branch must be used. The test case for this branch is defined in Table 2 in TTCN.

In line 1 of Table 2 , a set-request PDU is sent by the tester to the IUT. The constraint Set_request_base defines the initial contents of an instance of class Traffic Description Parameters. Line 3 represents the expected response from the IUT, i.e., a response PDU. The constraint of this response is $C$, defined in Table 1 , instantiated with parameter values. The request-id is 0 and the fourth parameter, the list of variable bindings, is empty. The received values of error-status and error-index are stored in parameters $p 2$ and $p 3$, respectively. In line 5 , if $p^{2}$ is equal to noError and $p 3$ is equal to 0 , a subtree called POSTAMBLE is attached in line 6. Line 7 defines the condition for failing the IUT. Lines 8 to 11 define the other events that can occur instead of a response PDU.

The purpose of the postamble in Table 3 is to check if the set operation has really been performed in the MIB. Line 2 sends a get-request PDU to the IUT. Its constraint is C1, defined in Table 5. Line 4 is for handling a response to the get-request from the IUT. Parameters of the response define the verdict of the test case. Line 6 defines the condition for passing the test. Line 7 fails the IUT if the opposite of the condition defined on line 


\begin{tabular}{|c|c|l|c|c|}
\hline $\mathrm{Nr}$ & Label & Behavior description & Cref \\
\hline 1 & & POSTAMBLE & & \\
2 & & !get_request & C1 \\
3 & & START transmissionTimer & \\
4 & LB & ?response & C(1, p2, p3, p4) \\
5 & & CANCEL transmissionTimer & \\
6 & & [p2=noError AND p3=0 AND p4=TrafficNoClpNoScrBinding2] & \\
7 & & [NOT (p2=noError AND p3=0 AND p4=TrafficNoClpNoScrBinding2] & \\
8 & & ?trap & PASS \\
9 & & -> LB & \\
10 & & ?TIMEOUT transmissionTimer & \\
11 & ?OTHERWISE & INCONC \\
\hline
\end{tabular}

Table 3: Postamble for the Creation of Traffic Description Parameters Test Case

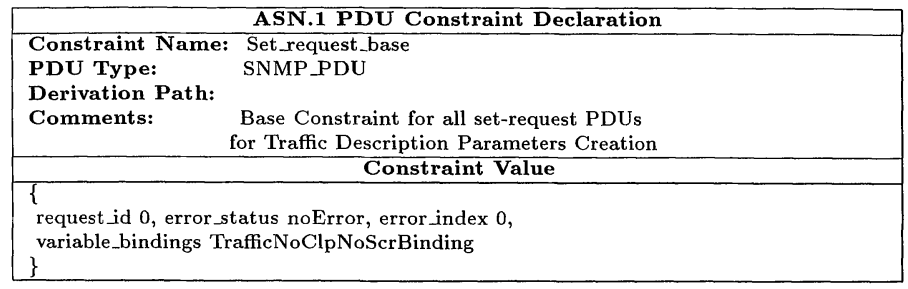

Table 4: Set Request Base Constraint

6 holds.

The constraint in Table 4 is the set-request PDU constraint.

Table 5 defines the constraint of the get-request PDU. In a get-request PDU, the variable binding list refers to an instance which has already been created, by the previous set-request PDU. The index value TSP_IUT_ParIndexVal designates the requested instance, represented as a row in a table, and the names of the requested columns are designated as unSpecified.

\section{CONCLUDING REMARKS}

We have developed a methodology for designing ATSs for testing the conformity of agents and managed objects, in managed nodes, to the MIB RFCs in the SNMP framework. In our approach, a class diagram representing classes of objects and their relations is developed. The dynamic behavior of each class is defined in SDL-92 through the concept of process type. ISO's conformance testing methodology is employed for the design of ATSs. In addition, we have identified how a MIB ATS can be structured and how some of the groups of test cases can be generated based on the SDL-92 specifications. Test cases in the ATS are specified in TTCN. An application has been made to the ATM MIB.

Use of an object-oriented specification language such as SDL-92 has several advantages. The specifications are more compact and also more readable because of non duplication of information. Test generation from these compact specifications is easier. However, the resulting test cases proved to be not so compact. This is because the test cases are designed for the instances while inheritance is on the types. The test cases need to take into account all the inherited features in the instances. Because ISO's test specification language TTCN integrates ASN.1, it was possible to specify precisely the data values in the test cases. A improvement that can be made to TTCN is the extension of constraint inheritance to ASN.1 values of type SEQUENCE OF, which are frequently required in MIB 
test cases.

Notifications (or traps) are spontaneous outputs of managed nodes. Presently, SMI conformance macros do not support notifications. More research is needed on the specification of notifications in MIBs and capture of implementation capabilities and their use in conformance test design.

Structure of ATSs need further improvements. These improvements could lead to new test groups for parameter variations and combinations. MIB integrity test cases are also left for further research.

Dependencies among MIB groups have an impact on individual test case design as well as on the overall ordering of the test cases in the ATSs. More research is needed in this direction.

\begin{tabular}{|c|}
\hline ASN.1 PDU Constraint Declaration \\
\hline $\begin{array}{ll}\text { Constraint Name: } & \text { C1 } \\
\text { PDU Type: } & \text { SNMP_PDU } \\
\text { Derivation Path: } & \\
\text { Comments: } & \text { get-request constraint for Traffic Descriptor Parameter Creation }\end{array}$ \\
\hline Constraint Value \\
\hline 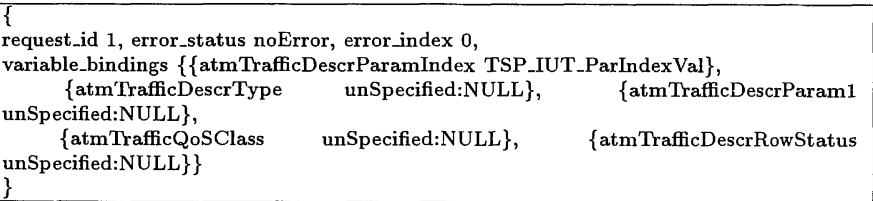 \\
\hline
\end{tabular}

Table 5: Get-Request Constraint

\section{References}

[1] M. Ahmed and K. Tesink. Definitions of Managed Objects for ATM Management Version 7.0, pages 1-90. IETF, March 1994.

[2] M. Barbeau and B. Sarikaya. Formal specification of MIBs. Technical report, University of Aizu, Aizu-Wakamatsu, Fukushima, Japan, 1995.

[3] G. Booch. Object Oriented Design with Applications. Benjamin / Cummings, 1994.

[4] CCITT. CCITT Specification and Description Language (SDL), pages 1-219. CCITT Recommendation Z.100, 1992.

[5] ATM Forum. ATM UNI Specification, Version 3.0. Prentice-Hall, 1993.

[6] ISO. ISO/IEC 8824 Specification of Abstract Syntax Notation-One(ASN.1).

[7] ISO. ISO/IEC 9646-1: Conformance Testing Methodology and Framework- Part 1: General Concepts, pages 1-31. ISO/IEC JTC1/SC21, 1991.

[8] ISO. ISO/IEC 9646-3: Conformance Testing Methodology and Framework- Part 3: The Tree and Tabular Combined Notation, pages 1-176. 1991.

[9] M.T. Rose. The Simple Book: An Introduction to Internet Management. Prentice Hall, Englewood Cliffs, New Jersey, second edition, 1994.

[10] B. Sarikaya. Principles of Protocol Engineering and Conformance Testing. Simon \& Schuster, September 1993.

Michel Barbeau got his Ph.D. in Computer Science from the University of Montreal, Canada in 1991. He joined the University of Sherbrooke in 1991 and works there as a professor. His research interests include development methods for telecommunication software.

Behcet Sarikaya got his Ph. D. from McGill University, Canada in 1984. He worked at the Universities of Sherbrooke, Concordia, Montreal in Canada and Bilkent in Turkey. He joined the University of Aizu, Japan in 1993 and works there as a professor. His research interests include multimedia networking. 